\title{
Clinical profile and long-term outcome of the first seizures in children
}

\author{
Pınar Arıcan ${ }^{1 \oplus}$, Hakan Salman ${ }^{2 \oplus}$, Nihal Olgaç Dündar ${ }^{3 \odot}$ \\ ${ }^{1}$ Department of Pediatric Neurology, Kahramanmaraş Necip Fazil Hospital, Kahramanmaraş; ${ }^{2}$ Department of Pediatrics, Süleyman \\ Demirel University, Isparta; ${ }^{3}$ Department of Pediatrics Neurology, İmir Katip Çelebi University, İmir, Turkey.
}

\begin{abstract}
Background. Seizures are one of the most common causes of pediatric admissions to hospitals in children. This study aims to identify the clinical profile and outcome of first seizures in children.

Methods. Children who presented to the pediatric neurology outpatient clinic and pediatric emergency service with a first-time seizure and aged one month through 18 years old were enrolled to the study. At the time of the study, enrolled children were categorized into three study groups according to seizure characteristics: febrile seizure, nonfebrile-provoked seizure and, unprovoked seizure.
\end{abstract}

Results. The study group consisted of 138 children. Of the 138 patients, 60 (43\%) had febrile first seizures, 23 $(17 \%)$ had nonfebrile-provoked first seizures, and $55(40 \%)$ had unprovoked first seizures. The patients did not experience the recurrence of a seizure by the treatment of underlying cause at the eighteenth month and the eighth year follow-up in the nonfebrile-provoked seizure group. Among the children admitted for unprovoked first seizures, $33(60 \%)$ patients had seizure recurrence during 18 month follow-up and 36 (82\%) patients had seizure recurrence during eight year follow-up. Seizure recurrence rate was statistically higher in patients with abnormal EEG and cranial MRI findings in the unprovoked seizure group $(\mathrm{p}<.05)$.

Conclusions. The patients with provoked first seizure did not develop epilepsy during eight year follow-up. However, 36 patients with unprovoked seizures were diagnosed with epilepsy during eight year follow-up. It is essential to determine the causes of the seizures and treat the condition.

Key words: children, epilepsy, outcome, risk factors, seizures.

A seizure is a common neurological symptom and one of the most frequent reasons for hospital admissions in children. ${ }^{1}$ It is suggested that approximately five percent of children will experience at least one episode of seizure in the first 16 years of life. The risk of seizures is higher in children less than three years of age and decreases with age. ${ }^{2,3}$

Seizures can be caused by an isolated event related to an acute situation, such as central nervous system infection, trauma, metabolic abnormality, toxic exposure, fever, or can occur

$凶$ Nihal Olgaç Dündar

nodundar@gmail.com

Received 5th September 2020, revised 22nd November 2020, 11th December 2020, accepted 24th December 2020. unprovoked in the absence of precipitating factors. ${ }^{4,5}$ Febrile seizures are the most common type of seizure seen in the pediatric population, especially in children younger than five years of age. ${ }^{6,7}$

While a seizure is defined as the occurrence of signs or symptoms due to abnormal or synchronous electrical activity in the brain, there is a paucity of data regarding acute seizures in children. In this study, we aimed to identify the clinical profile and outcome of first seizures in children.

\section{Material and Methods}

This prospective cross-sectional study was performed on children who were admitted with a history of seizure, to the pediatric neurology 
outpatient clinic and pediatric emergency service from January 1, 2010 to December 31, 2010. Demographic data, clinical reports, laboratory data, cranial magnetic resonance imaging (MRI), and electroencephalography (EEG) results of the included patients were recorded and reviewed. The study protocol was approved by the Clinical Research Ethics Committee of Süleyman Demirel University (number: 2010/19-7). Informed consent was obtained from the parents.

Children who presented to the pediatric neurology outpatient clinic and pediatric emergency service with a first-time seizure and aged one month through 18 years old were enrolled to study. Patients were excluded in the study if they had received any antiepileptic drug or had been diagnosed with epilepsy. In each patient the following laboratory tests were obtained: white blood count, C-reactive protein, serum electrolytes, and blood sugar. Electroencephalography was performed in all patients and cranial MRI was performed wherever indicated.

Patients were followed up in our pediatric neurology outpatient clinic. At the time of the study enrollment, children were categorized into three study groups according to seizure characteristics: febrile seizure, nonfebrileprovoked seizure, and unprovoked seizure. The data on seizure types were collected by definitions of parents. The seizure types were classified according to the International League Against Epilepsy classification of seizure types and the epilepsies. ${ }^{8} \mathrm{~A}$ febrile seizure was classified as complex febrile seizure if it is focal, prolonged (with a duration of $>15$ minutes), or recurrent within 24 hours. $^{7}$ During 18 months follow-up, the patients were clinically examined every three months. For the long-term followup, data on recurrent seizures were collected by interviews of guardians by telephone in the eighth year.

Statistical analysis was performed using Statistical Package for the Social Sciences software program version 21.0. Categorical variables were summarized using percentages. Continuous variables were summarized using means. The Chi-square test and Fisher's exact test were used for comparison between independent groups of categorical data. For all statistical tests, values of $\mathrm{p}<0.05$ (two-tailed) were considered statistically significant.

\section{Results}

A total of 138 children were enrolled and evaluated of whom $55(40 \%)$ were females and $83(60 \%)$ males, the mean age was 42 months. In total, 126 (91\%) patients had generalized seizures and 12 (9\%) patients had focal seizures. Cranial MRI was obtained in 110 patients and was normal in $88(80 \%)$ patients. Electroencephalography was obtained in 138 patients and was normal in 105 (76\%) patients. Of the 138 patients, 60 (43\%) had febrile first seizures, $23(17 \%)$ had nonfebrile-provoked first seizures, and $55(40 \%)$ had unprovoked first seizures (Table I). Out of a total of 108 patients, $78(72 \%)$ patients had seizure recurrence during eight year follow-up.

Table I. Demographic characteristics and clinical features of patients presenting with first seizure.

\begin{tabular}{lc}
\hline Gender & \\
Female & $55(40 \%)$ \\
Male & $83(60 \%)$ \\
Age (months)(means \pm SD) & 42 months \\
Seizure type & \\
$\quad$ Generalized & $126(91 \%)$ \\
Focal & $12(9 \%)$ \\
Cranial MRI & \\
$\quad$ Normal & $88(80 \%)$ \\
$\quad$ Abnormal & $22(20 \%)$ \\
EEG findings, n (\%) & \\
$\quad$ Normal & $105(76 \%)$ \\
Abnormal & $33(24 \%)$ \\
Seizure characteristics & \\
Febrile seizure & $60(43 \%)$ \\
Nonfebrile-provoked seizure & $23(17 \%)$ \\
Unprovoked seizure & $55(40 \%)$ \\
\hline MRI: magnetic resonance imaging, EEG: & \\
electroencephalogram. &
\end{tabular}




\section{Febrile first seizures}

Among the children admitted for febrile first seizures during the study period, 13 patients had complex febrile seizures and 47 patients had simple febrile seizures. The mean age was 24 months. During 18 month follow-up, 28 (47\%) patients with febrile first seizures had seizure recurrence. Of 28 patients, 19 had complex febrile seizures and 9 had simple febrile seizures. At eight year follow-up, among all 55 patients, $22(40 \%)$ patients had no seizure recurrence. A total of $33(60 \%)$ patients had febrile seizure recurrence at eight year follow-up (Table II). Of 33 patients, 21 had complex febrile seizures and 11 had simple febrile seizures.

\section{Nonfebrile-provoked first seizures}

Among the children admitted for provoked first seizures during the study period, 12 patients had central nervous system infection, six patients had head trauma, three patients had hyponatremia, one patient had carbon monoxide poisoning, one patient had hypocalcemia and hypomagnesemia. The patient who had both hypocalcemia and hypomagnesemia was found to carry a homozygous mutation in the TRPM6 gene. During 18 month follow-up, patients with nonfebrile-provoked first seizures had no seizure recurrence. At the eight year follow-up, nine patients still had no seizure recurrence.

\section{Unprovoked first seizures}

Among the children admitted for unprovoked first seizures during the study period, $22(40 \%)$ patients had only one seizure and $33(60 \%)$ patients had seizure recurrence during 18 month follow-up. At eight year follow-up, in a total of 44 patients, eight patients (18\%) still had no seizure recurrence and three patients with a single seizure had seizure recurrence. Thirty-six $(82 \%)$ patients had seizure recurrence at eight year follow-up. Twenty-one (78\%) patients with abnormal EEG findings had seizure recurrence at 18 month follow-up. Thirteen (81\%) patients with abnormal cranial MRI findings such as ischemic gliosis and mesial temporal sclerosis had seizure recurrence during 18 month followup. Seizure recurrence rates were statistically higher in patients with abnormal EEG and cranial MRI findings ( $p$ <.05), however, no statistically significant differences were observed according to the seizure types (Table III). There were no significant differences in febrile and unprovoked first seizures for seizure recurrence rate at 18 month follow-up ( $>$ >05), however, the seizure recurrence rate was statistically higher in patients with unprovoked first seizures at eight year follow-up $(p<.05)$ (Table II).

\section{Discussion}

In the literature, clinical and outcome profiles of childhood seizures can be different in limited reported data. ${ }^{9,10}$ In this study, we explored the clinical spectrum and long-term outcome of children who were admitted to the hospital with a history of first seizure.

First-line investigations should include tests of blood glucose level and serum electrolytes. In patients with the suspected toxic or druginduced etiology, if possible blood levels of the suspected drug must be measured. ${ }^{11}$ In our study, the following laboratory tests were

Table II. Seizure recurrence rate of the first seizures in children.

\begin{tabular}{lccc}
\hline & Febrile seizure group & Unprovoked seizure group & p value \\
\hline Seizure recurrence at 18 month follow up & & & \\
Yes & $28(47 \%)$ & $33(60 \%)$ & 0.191 \\
No & $32(53 \%)$ & $22(40 \%)$ & \\
Seizure recurrence at 8 year follow up & & & \\
$\quad$ Yes & $33(60 \%)$ & $36(82 \%)$ & 0.027 \\
No & $22(40 \%)$ & $8(18 \%)$ & \\
\hline
\end{tabular}


Table III. Comparison between seizure free and seizure recurrence groups in patients with unprovoked first seizures.

\begin{tabular}{lccc}
\hline & \multicolumn{2}{c}{ Seizure recurrence } & \multirow{2}{*}{ p value } \\
\cline { 2 - 3 } & Yes & No & \multirow{2}{*}{0.437} \\
\hline Gender & $20(64 \%)$ & $11(36 \%)$ & \\
$\quad$ Female & $13(54 \%)$ & $11(46 \%)$ & 0.876 \\
$\quad \begin{array}{l}\text { Male } \\
\text { Family history of epilepsy }\end{array}$ & $5(62 \%)$ & $3(38 \%)$ & \\
$\quad$ Yes & $28(60 \%)$ & $19(40 \%)$ & 0.141 \\
$\quad$ No & & & \\
Seizure type & $29(57 \%)$ & $22(43 \%)$ & 0.039 \\
$\quad$ Generalized & $4(100 \%)$ & $0(0 \%)$ & \\
$\quad$ Focal & $20(51 \%)$ & $19(49 \%)$ & 0.004 \\
Cranial MRI & $13(81 \%)$ & $3(19 \%)$ & \\
$\quad$ Normal & & $17(60 \%)$ & \\
$\quad$ Abnormal & $11(40 \%)$ & $6(22 \%)$ & \\
EEG findings, n (\%) & $21(78 \%)$ & & \\
$\quad$ Normal & & & \\
$\quad$ Abnormal & & & \\
\hline
\end{tabular}

EEG: Electroencephalogram

obtained for each patient: white blood count, C-reactive protein, serum electrolytes, and blood sugar. We found that $23(17 \%)$ patients who had nonfebrile-provoked first seizures did not experience recurrence of a seizure by the treatment of underlying cause at eighteen month and eight year follow-up. It is essential to determine the causes of the seizures, and treat the condition.

Electroencephalography and cranial MRI are useful tools in the diagnosis of epilepsy, identification of a specific syndrome, and prediction of long-term outcome. ${ }^{12,13}$ The rates of epileptiform discharges varied from 44 to $80 \%$ in children who were admitted with seizures in reported studies. ${ }^{14-16}$ In our study, EEG was abnormal in $24 \%$ (33) patients. The seizure recurrence rate was statistically higher in patients with abnormal EEG and cranial MRI findings. It is estimated that recurrence risk was significantly less among the patients with normal EEG and cranial MRI when compared to those with abnormal EEG and cranial MRI.

Population-based studies indicate that acute symptomatic seizures represent $40-50 \%$ of all cases of seizures. ${ }^{12,17}$ Sartori et al. ${ }^{18}$ reported that $32.5 \%$ of patients with acute symptomatic seizures experienced seizure recurrence at 4-year follow-up. In our study, 83 (60\%) patients presented with acute symptomatic seizures. Seizures associated with reversible metabolic or toxic disturbances are associated with a minor risk of subsequent epilepsy.,19 In our study, none of the patients with metabolic or toxic disturbances developed epilepsy at eight year follow-up. Approximately one-third of children with a first febrile seizure will experience a recurrence, and $10 \%$ will have three or more febrile seizures. ${ }^{20,21}$ Our study showed that $60 \%$ of patients who were admitted for febrile first seizures had febrile seizure recurrence at eight year follow-up.

The first unprovoked seizure has a recurrence risk of 30-50\%, however, the second unprovoked seizure has a recurrence risk of 70$80 \%$, justifying the diagnosis of epilepsy. ${ }^{5}$ In a systematic review and meta-analysis by Garcia Pierce et al. $^{22}$, it estimated a recurrence rate within 3 years of $45 \%$ after a first unprovoked seizure. Sartori et al. ${ }^{18}$ reported that $60 \%$ of 
patients with unprovoked seizures experienced seizure recurrence over the four-year followup. The strongest risk factors for recurrence of unprovoked seizures are EEG abnormalities, family history of epilepsy, and pre-existing static brain abnormalities. ${ }^{23,24}$ In our study, the febrile seizures were the most common type of first seizures, respectively followed by unprovoked seizures and nonfebrile-provoked seizures. The patients with provoked first seizure did not develop epilepsy at eight year followup. However, 36 patients with unprovoked seizures were diagnosed with epilepsy at eight year follow-up.

Our study has some limitations. The data on seizure types were collected by definitions of parents. Also, we could not make detailed investigations about possible underlying etiologies of first unprovoked seizures due to the limited resources at that period in 2010. Prospective studies are needed to better define the clinical profile and outcome of first seizures in children.

Ideally, the goals in the management of acute seizures are to stabilize the patient, identify any electrolyte imbalance and hypoglycemia, terminate the ongoing seizure activity as soon as possible, determine the underlying etiology, and decide on the need of long-term antiepileptic drugs. Misdiagnosis carries the potential risk of legal problems, can cause family anxiety and lead to an excessive hospital stay. The most important factor in diagnosing seizures is to rule out the possibility of a nonepileptic event. It can be useful to record the seizure-like events with a digital or video camera by the patient's parents. Investigations should include prompt EEG. It is important to delineate a detailed description of the clinical seizure for the correct diagnosis, treatment, and prognosis.

\section{Acknowledgment}

There was no assistance or efforts beyond those of the primary authors. This work has not been presented or published elsewhere.

\section{Author contribution}

The authors confirm contribution to the paper as follows: study conception and design: NOD; data collection: HS; analysis and interpretation of results: NOD, PA, HS; draft manuscript preparation: NOD, PA. All authors reviewed the results and approved the final version of the manuscript.

\section{Ethical approval}

The study protocol was approved by the Clinical Research Ethics Committee of Süleyman Demirel University (number: 2010/19-7).

\section{Source of funding}

The authors received no financial support for this research, authorship, and or publication of this article.

\section{Conflicts of interest}

The authors declare no potential conflicts of interest regarding this research, authorship, and/or publication of this article.

\section{REFERENCES}

1. Friedman MJ, Sharieff GQ. Seizures in children. Pediatr Clin North Am 2006; 53: 257-277.

2. Hauser WA. The prevalence and incidence of convulsive disorders in children. Epilepsia 1994; 35(Suppl 2): S1-S6.

3. Ojha AR, Aryal UR. Clinico-etiological profile of children with seizures admitted in a tertiary centre. J Kathmandu Med Coll 2015; 4: 55-58.

4. Nowacki TA, Jirsch JD. Evaluation of the first seizure patient: key points in the history and physical examination. Seizure 2017; 49: 54-63.

5. Pohlmann-Eden B, Beghi E, Camfield C, Camfield P. The first seizure and its management in adults and children. BMJ 2006; 332: 339-342.

6. Almojali AI, Ahmed AE, Bagha MY. Prognostic factors for epilepsy following first febrile seizure in Saudi children. Ann Saudi Med 2017; 37: 449-454. 
7. Seinfeld S, Shinnar S. Febrile seizures. In: Swaiman KF, Ashwal S, Ferriero DM, et al (eds). Swaiman's Pediatric Neurology: Principles and Practice (6th ed) Philadelphia: Elsevier, 2017: 519-523.

8. Berg AT, Berkovic SF, Brodie MJ, et al. Revised terminology and concepts for organization of seizures and epilepsies: report of the ILAE Commission on Classification and Terminology, 2005-2009. Epilepsia 2010; 51: 676-685.

9. Geerts A, Arts WF, Stroink H, et al. Course and outcome of childhood epilepsy: a 15-year followup of the Dutch study of epilepsy in childhood. Epilepsia 2010; 51: 1189-1197.

10. Ashmawi A, Hosny H, Abdelalim A, Bianchi E, Beghi E. The long-term prognosis of newly diagnosed epilepsy in Egypt: a retrospective cohort study from an epilepsy center in Greater Cairo. Seizure 2016; 41: 86-95.

11. Sasidaran K, Singhi S, Singhi P. Management of acute seizure and status epilepticus in pediatric emergency. Indian J Pediatr 2012; 79: 510-517.

12. Narayanan J, Murthy JMK. New-onset acute symptomatic seizure in a neurological intensive care unit. Neurol India 2007; 55: 136-140.

13. Debicki DB. Electroencephalography after a single unprovoked seizure. Seizure 2017; 49: 69-73.

14. Martin ET, Kerin T, Christakis DA, et al. Redefining outcome of first seizures by acute illness. Pediatrics 2010; 126: e1477-e1484.

15. Adhikari S, Sathian B, Koirala DP, Rao KS. Profile of children admitted with seizures in a tertiary care hospital of Western Nepal. BMC Pediatr 2013; 13: 43.
16. Poudel P, Parakh P, Mehta K. Clinical profile, aetiology and outcome of afebrile seizures in children. JNMA J Nepal Med Assoc 2013; 52: 260266.

17. Beleza P. Acute symptomatic seizures: a clinically oriented review. Neurologist 2012; 18: 109-119.

18. Sartori S, Nosadini M, Tessarin G, et al. First-ever convulsive seizures in children presenting to the emergency department: risk factors for seizure recurrence and diagnosis of epilepsy. Dev Med Child Neurol 2019; 61: 82-90.

19. Gulati S, Kaushik JS. How I treat a first single seizure in a child. Ann Indian Acad Neurol 2016; 19: 29-36.

20. Hesdorffer DC, Shinnar S, Lax DN, et al; FEBSTAT Study Team. Risk factors for subsequent febrile seizures in the FEBSTAT study. Epilepsia 2016; 57: 1042-1047.

21. Al-Eissa YA. Febrile seizures: rate and risk factors of recurrence. J Child Neurol 1995; 10: 315-319.

22. Garcia Pierce J, Aronoff S, Del Vecchio, M. Systematic review and meta-analysis of seizure recurrence after a first unprovoked seizure in 815 neurologically and developmentally normal children. J Child Neurol 2017; 32: 1035-1039.

23. Schreiner A, Pohlmann-Eden B. Value of the early electroencephalogram after a first unprovoked seizure. Clin Electroencephalogr 2003; 34: 140-144.

24. Berg AT. Risk of recurrence after a first unprovoked seizure. Epilepsia 2008; 49: 13-18. 